\title{
Radiation Induced Mutation for Resistance against Dry Root Rot in Chickpea (Cicer arietinum Linn.)
}

\author{
Maruti Pawar*, O.M. Gupta and Devashish Chobe \\ Department of Plant Pathology, JNKVVV, Jabalpur (M.P), India \\ *Corresponding author
}

\begin{tabular}{|c|}
\hline Keywords \\
\hline $\begin{array}{l}\text { Rhizoctonia } \\
\text { bataticola, Gamma- } \\
\text { ray, EMS, Cicer } \\
\text { arietinum L }\end{array}$ \\
\hline Article Info \\
\hline $\begin{array}{l}\text { Accepted: } \\
\text { 26 June } 2018 \\
\text { Available Online: } \\
\text { 10 July } 2018\end{array}$ \\
\hline
\end{tabular}

A B S T R A C T

Chickpea (Cicer arietinum L.) is an important cool-season food legume grown extensively by the poor farmers throughout the Indian subcontinent. In India chickpea is being grown in 8.32 million hectare with production of 9.8 million tones and $925-\mathrm{kg} / \mathrm{ha}$ productivity (Project coordinators report, 2014-15). The Dry root rot (DRR) of chickpea caused by necrotropic fungus Rhizoctonia bataticola. During the past few decades, modern techniques such as mutation breeding by radiation and chemical mutagens and genetic engineering methodology have been tried to develop resistant cultivars of many crop plants. The utilization of mutation breeding is a simple, less cost full and time saving method. Present investigation entitled "Radiation induced mutation for resistance against Rhizoctonia bataticola in chickpea (Cicer arietinum Linn.)" was aimed at identification of suitable mutant or a combination of mutants influencing resistance to dry root rot in chickpea. The experimental material was consisted of the population of three selected cultivars of chickpea (JG 63, JG 74, and JG 130) grown in randomized complete block design in the Seed Breeding Farm, Department of Plant Breeding and Genetics, College of Agriculture, Jabalpur (M.P) under AICRP on chickpea project during Rabi 2014-15. Dry seeds (10-12\% moisture content) of these varieties were irradiated with different doses of gamma rays $(150 \mathrm{~Gy}, 200 \mathrm{~Gy}$ and $400 \mathrm{GY})$. Another set of presoaked seeds in distilled water $(12 \mathrm{hrs}$.) were treated with ethyl methane sulphonate at different concentration $(0.3$, 0.4 and $0.5 \%$ ) prepared for $6 \mathrm{hrs}$. A portion of seeds irradiated at 150 and $200 \mathrm{GY}$ gammaray doses were also treated with $0.3 \%$ and $0.4 \%$ EMS independently for 6 hrs. present findings revealed that JG 63, JG 74, JG 130 showed significant reaction for mutagenic treatments i.e, 200Gy, 400Gy, 0.3\% EMS, 0.4\% EMS, 150Gy+0.3\% EMS, 200Gy+0.3\% EMS. Among 11 treatments, 7 have shown effect on biological traits of experimental genotypes i.e. change in seedling height, decrease in germination percentage and decrease in plant height as compared to control.

\section{Introduction}

Chickpea is a cool season pulse crop and is grown in several countries worldwide as a food source. Chickpea is the third most important food legume crop and India is the largest producer contributing to $65 \%$ of world's chickpea production. The improvement of chickpea using conventional breeding approaches has been hampered due 
to lack of sufficient genetic variability. Mutagenesis is a common and efficient tool to create new desirable genetic variability in chickpea [1]. The use of ionizing radiation such as, X-rays, gamma rays, and neutrons and chemical mutagens for inducing variation is well established. Induced mutation have been used to improve major crop which are seed propagated. Mutation can be linked to changes in DNA sequences for some plant traits and to establish molecular maps in structural and functional genomics of crop plants. These in turn would lead to a rapid enhancement of crop yields and quality [2].

\section{Materials and Methods}

The experiment was carried out under AICRPChickpea, at seed breeding farm, Adhartal, JNKVV, Jabalpur (M.P.) during rabi 2014-15. Temperature extremes vary between minimum temperature of $2^{\circ} \mathrm{C}$ in December and January months to maximum temperature of $45^{\circ} \mathrm{C}$ in May and June months. The average annual rainfall mostly received between mid - June to first week of October with occasional showers in limited quantum during the winter months, ranges between $1000-1500 \mathrm{~mm}$. The relative humidity remains minimum 20 to $35 \%$ during summer and medium 40 to $60 \%$ during winter season, while it attains maximum values of 80 to $95 \%$ during rainy season. Chickpea seeds of JG 130, JG 63, and JG 74 entries/ varieties were collected from healthy plants at maturity stage of the crop from AICRP on chickpea located at seed breeding farm J.N.K.V.V., Jabalpur. Gamma irradiation has been performed (Nuclear Research Laboratory IARI, New Delhi) in gamma chamber by exposing the seeds to the gamma rays from ${ }^{60} \mathrm{Co}$ source was filled and handpicked uniform sized seeds with moisture content of $10-12 \%$ were chosen for irradiation. A sample of 120 seeds pre treatment will be packed in butter paper cover and placed in 100 curie ${ }^{60} \mathrm{Co}$ gamma cells the treatments will be given for various duration depending on the doses required (150Gray, 200Gray and 400 Gray) with the dose rate of say $54.05 \mathrm{rads} / \mathrm{sec}$. Set of presoaked seeds of selected genotypes in distilled water (12 hrs.) were treated with ethyl methane sulphonate at different concentration by using magnetic stirrer $(0.3,0.4$ and $0.5 \%)$ for $6 \mathrm{hrs}$. with constant intermitted shaking in shaker and after that washed under running tap water. A portion of seeds irradiated at 150 and 200 GY gamma- ray doses were also treated with 0.3 and 0.4 EMS independently for $6 \mathrm{hrs}$ with constant intermitted shaking in shaker and after that washed under running tap water. A total of 11-treatment combinations (including control) were evaluated separately for each variety planted in Randomized Block Design with three replication following biological parameters of observation during Rabi season 2014-15 at Seed Breeding farm, JNKVV Jabalpur.

\section{Results and Discussion}

Impact of the mutagenic treatments on seed germination and some morphological parameters on chickpea variety JG 63

Considering the value of control as $100 \%$ for all the morphological traits, the data are presented (Table 1).

\section{Germination (\%)}

The mean seed germination percentage was reported to be $84.36 \%$ at $150 \mathrm{~Gy}, 72.14 \%$ at 200Gy, $64.50 \%$ at $400 \mathrm{~Gy}$ respectively as compared to control. Similarly, the germination (\%) was also reduced by using chemical mutagens. The germination (\%) was noted to be $68.70 \%$ at $0.2 \%$ EMS, $60.31 \%$ at $0.3 \%$ EMS and $52.67 \%$ at $0.4 \%$ EMS treatments respectively. Whereas, the mean seed germination percentage in combination of Gamma irradiation and EMS at treatment was reported as $66.41 \%$ at $150 \mathrm{GY}+0.2 \% \mathrm{EMS}$ 
and $54.49 \%$ at $200 \mathrm{~Gy}+0.2 \%$ EMS respectively as compared to control.

\section{Plant height}

The minimum mean plant height (\%) i.e. was noted $71.98 \%$ at $200 \mathrm{~Gy}, 75.46 \%$ in $0.3 \%$ EMS and $79.15 \%$ in combination treatment $200 \mathrm{~Gy}+0.3 \%$ EMS respectively, among the all treatments as compared to control.

\section{Number of pods per plants}

The maximum number of pods per plant (\%) i.e, $90.20 \%$ was noted at $150 \mathrm{~Gy}$, followed by $67.00 \%$ at $200 \mathrm{~Gy}$ and $60.82 \%$ at $400 \mathrm{~Gy}$. Minimum number of pods per plant (\%) was also increased using chemical mutagens, the highest pods per plant (\%) in EMS treatment was noted at $0.3 \%$ EMS (66.49\%), whereas in combination treatment highest mean pods per plant (\%) was reported to be $75.77 \%$ at $150 \mathrm{GY}+0.2 \%$ EMS as compared to control.

\section{Number of seeds per plant}

The mean seeds per plant $(\%)$ were noted to be $75.61 \%$ at $150 \mathrm{~Gy}, 62.61 \%$ at $200 \mathrm{~Gy}$ and $54.46 \%$ at 400 Gy respectively. Similarly in chemical mutagenic treatments the mean seeds per plant (\%) were noted to be $74.80 \%$ at $0.2 \%$ EMS, $40.98 \%$ at $0.3 \%$ EMS and $80.49 \%$ at $0.4 \%$ EMS respectively. In the combination treatment highest number of mean seeds per plant (\%) among all the mutagenic treatments was found at $200 \mathrm{~Gy}+0.3 \%$ EMS (84.56\%) as compared to control.

\section{Seed yield per plant}

The mean seed yield per plant (\%) was noted to be $85.52 \%$ at $200 \mathrm{~Gy}, 63.13 \%$ at $150 \mathrm{~Gy}$ and $60.15 \%$ at $400 \mathrm{~Gy}$.

Similarly in chemical mutagenic treatments the highest percentage of mean seed yield per plant was $76.12 \%$ at $0.2 \%$ EMS, whereas in combination treatments $(200 \mathrm{GY}+0.2 \%$ EMS $)$ the highest percentage of mean seed yield per plant was noted to be $70.75 \%$ respectively.

\section{Seedling height}

Mean seedling height (\%) after 15 days of sowing was reported to be $47.30 \%$ at $150 \mathrm{~Gy}$, $43.25 \%$ at $200 \mathrm{~Gy}$ and $22.90 \%$ at $400 \mathrm{~Gy}$ respectively. Similarly in chemical mutagenic treatments the seedling height was $75.56 \%$ at $0.2 \%$ EMS, $61.68 \%$ at $0.3 \%$ EMS and $51.66 \%$ at $0.4 \%$ EMS respectively. Whereas in combination treatments the mean seedling height was noted to be $87.36 \%$ at $150 \mathrm{~Gy}+0.2 \% \mathrm{EMS}, \quad 86.58 \%$ at $200 \mathrm{~Gy}+0.2 \% \mathrm{EMS}, \quad \quad 75.02 \%$ at $150 \mathrm{~Gy}+0.3 \% \mathrm{EMS}$ and $48.80 \%$ at $200 \mathrm{~Gy}+$ $0.3 \%$ EMS respectively, as compared to control.

\section{0 seed weight}

The mean 100 seed weight (\%) was reported to be $84.74 \%$ at $150 \mathrm{~Gy}, 89.78 \%$ at $200 \mathrm{~Gy}$ and $81.10 \%$ at $400 \mathrm{~Gy}$, respectively. Whereas, in chemical mutagenic treatments it was recorded to be $77.79 \%$ at $0.2 \%$ EMS, $76.61 \%$ at $0.3 \%$ EMS and $80.74 \%$ at $0.4 \%$ EMS also in combination treatments the mean 100 seed weight percentage was noted to be $73.24 \%$ at $150 \mathrm{~Gy}+0.2 \% \mathrm{EMS}, 78.15 \%$ at $200 \mathrm{~Gy}+0.2 \%$ EMS, $78.56 \%$ at $150 \mathrm{~Gy}+0.3 \%$ EMS and $74.01 \%$ at $200 \mathrm{~Gy}+0.3 \%$ EMS respectively, as compared to control.

\section{Lethality}

The highest lethality percentage was recorded in combination treatments, which was $71.93 \%$ at $200 \mathrm{~Gy}+0.3 \% \mathrm{EMS}$, whereas in physical mutagenic treatment the maximum lethality was recorded to be $53.05 \%$ in $400 \mathrm{~Gy}$ and in chemical mutagenic treatments the maximum lethality percentage was recorded to be $61.66 \%$ at $0.4 \%$ EMS as compared to control. 
Impact of the mutagenic treatments on seed germination and some morphological parameters on chickpea variety JG 74

Considering the value of control $100 \%$ for all the morphological traits, the data are presented and discussed (Table 2).

\section{Germination (\%)}

The mean seed germination percentage was reported to be $84.62 \%$ at $150 \mathrm{~Gy}, 64.53 \%$ at $200 \mathrm{~Gy}$ and $53.42 \%$ at $400 \mathrm{~Gy}$ respectively as compared to control.

Similarly, using chemical mutagens also reduced the germination $\%$ and it was found $67.09 \%$ at $0.2 \%$ EMS, $56.41 \%$ at $0.3 \%$ EMS and $54.27 \%$ at $0.4 \%$ EMS treatments respectively.

Whereas, the mean seed germination percentage in combination of Gamma irradiation and EMS at treatment were noted to be $70.09 \%$ at $150 \mathrm{GY}+0.2 \%$ EMS and 58.97 $\%$ at $200 \mathrm{~Gy}+0.2 \% \mathrm{EMS}$ respectively as compared to control.

\section{Plant height}

The mean plant height (\%) was noted to be $59.11 \%$ at $200 \mathrm{~Gy}, 90.06 \%$ at $0.3 \%$ EMS and $79.49 \%$ in combination treatment of $200 \mathrm{~Gy}+0.3 \%$ EMS respectively, which was minimum among the all treatments as compared to control.

\section{Number of pods per plants}

The highest number of mean pods per plant (percentage) were reported to be $90.95 \%$ at $150 \mathrm{~Gy}$ and $56.37 \%$ at $200 \mathrm{~Gy}$ respectively, whereas in combination treatment highest mean pods per plant percentage was reported to be $78.19 \%$ at $150 \mathrm{GY}+0.2 \%$ EMS as compared to control.

\section{Number of seeds per plant}

The mean seeds per plant percentage were noted to be $89.99 \%$ at $150 \mathrm{~Gy}, 74.29 \%$ at $200 \mathrm{~Gy}$ and $40.71 \%$ at $400 \mathrm{~Gy}$. Similarly in chemical mutagenic treatments the mean seeds per plant percentage were noted to be $97.86 \%$ at $0.2 \%$ EMS, $36.43 \%$ at $0.3 \%$ EMS and $63.57 \%$ at $0.4 \%$ EMS respectively. Whereas the combination treatment showed highest mean seeds per plant percentage among the all mutagenic treatments which was noted to be $89.99 \%$ at $200 \mathrm{~Gy}+0.3 \%$ EMS as compared to control.

\section{Seed yield per plant}

The mean seed yield per plant percentage was noted to be $82.58 \%$ at $150 \mathrm{~Gy}, 67.50 \%$ at $200 \mathrm{~Gy}, 52.60 \%$ at $400 \mathrm{~Gy}$ and $83.25 \%$ at $0.2 \%$ EMS, whereas in combination treatments the highest percentage of mean seed yield per plant was noted to be $71.52 \%$ at $150 \mathrm{~Gy}+0.2 \% \mathrm{EMS}$ treatment, followed by $70.35 \%$ at $200 \mathrm{GY}+0.3 \%$ EMS respectively.

\section{Seedling height}

Mean seedling height percentage after 15 days of sowing was reported to be $77.05 \%$ at $150 \mathrm{~Gy}, 96.97 \%$ at $200 \mathrm{~Gy} 82.35 \%$ at $400 \mathrm{~Gy}$, $74.24 \%$ at $0.2 \% \mathrm{EMS}, 76.29 \%$ at $0.3 \% \mathrm{EMS}$ and $84.62 \%$ at $0.4 \%$ EMS, respectively. Whereas, in combination treatments the mean seedling height was noted to be $83.56 \%$ at $150 \mathrm{~Gy}+0.2 \% \mathrm{EMS}, 87.12 \%$ at $200 \mathrm{~Gy}+0.2 \%$ EMS, $92.20 \%$ at $150 \mathrm{~Gy}+0.3 \% \mathrm{EMS}$ and $48.80 \%$ at $200 \mathrm{~Gy}+0.3 \%$ EMS, respectively, as compared to control.

\section{0 seed weight}

The mean 100 seed weight $(\%)$ was reported to be 78.74 at $150 \mathrm{~Gy}, 82.52 \%$ at $200 \mathrm{~Gy}$ $75.19 \%$ at $400 \mathrm{~Gy}, 76.96 \%$ at $0.2 \% \mathrm{EMS}$, $90.55 \%$ at $0.3 \%$ EMS and $89.60 \%$ at 
0.4\%EMS, respectively. In combination treatments the mean 100 seed weight percentage was noted to be $92.73 \%$ at $150 \mathrm{~Gy}+0.2 \% \mathrm{EMS}, \quad 81.69 \%$ at $200 \mathrm{~Gy}+0.2 \% \mathrm{EMS}, \quad 77.20 \%$ at $150 \mathrm{~Gy}+0.3 \% \mathrm{EMS}$ and $81.10 \%$ at 200Gy+ $0.3 \%$ EMS as compared to control.

\section{Lethality}

The highest lethality percentage was recorded in physical mutagenic treatments, which was $55.00 \%$ at $150 \mathrm{~Gy}$, whereas in chemical mutagenic treatment the maximum lethality was recorded to be $43.61 \%$ at $0.2 \%$ EMS. Similarly, in combination treatments the maximum lethality percentage was recorded to be $45.56 \%$ at $150 \mathrm{~Gy}+0.2 \%$ EMS as compared to control.

Impact of the mutagenic treatments on seed germination and some morphological parameters on chickpea variety JG 130

Considering the value of control as $100 \%$ for all the morphological traits, the data are presented and discussed (Table 3).

\section{Germination (\%)}

The mean seed germination percentage was reported to be $70.11 \%$ at $150 \mathrm{~Gy}, 60.92 \%$ at $200 \mathrm{~Gy}, 56.32 \%$ at $400 \mathrm{~Gy}, 58.62 \%$ at $0.2 \%$ EMS, $49.43 \%$ at $0.3 \%$ EMS and $43.68 \%$ at $0.4 \%$ EMS treatments, respectively. Whereas, the mean seed germination percentage in combination of Gamma irradiation and EMS at treatment were reported $58.62 \%$ at $150 \mathrm{GY}+0.2 \%$ EMS and $48.28 \%$ at $200 \mathrm{~Gy}+0.2 \% \mathrm{EMS}$ respectively as compared to control.

\section{Plant height}

The minimum mean plant height $(\%)$ was noted to be $67.10 \%$ at $400 \mathrm{~Gy}, 84.01 \%$ at $0.4 \%$ EMS and $69.73 \%$ in combination treatment
$(200 \mathrm{~Gy}+0.3 \%)$ EMS respectively, compared to control.

\section{Number of pods per plants}

The number of pods per was reported to be to be $61.57 \%$ at $150 \mathrm{~Gy}, 35.57 \%$ at $400 \mathrm{~Gy}$, $28.19 \%$ at $200 \mathrm{~Gy}$ and $61.57 \%$ at $0.4 \%$ EMS. Whereas, in the combination treatment highest mean pods per plant (percentage) was reported to be $85.22 \%$ at $150 \mathrm{GY}+0.2 \%$ EMS as compared to control.

\section{Number of seeds per plant}

The mean seeds per plant (percentage) were noted to be $56.6 \%$ at $150 \mathrm{~Gy}, 54.05 \%$ at $200 \mathrm{~Gy}$ $58.57 \%$ at $400 \mathrm{~Gy}, 85.59 \%$ at $0.2 \%$ EMS, $53.16 \%$ at $0.3 \%$ EMS and $64.14 \%$ at $0.4 \%$ EMS, respectively. Whereas, the combination treatment $(200 \mathrm{~Gy}+0.3 \%$ EMS $)$ showed highest mean seeds per plant percentage among the all mutagenic treatments which was noted to be $91.00 \%$ as compared to control.

\section{Seed yield per plant}

The mean seed yield per plant (percentage) was noted to be $61.93 \%$ at $150 \mathrm{~Gy}, 73.65 \%$ at $200 \mathrm{~Gy}, 59.00 \%$ at $400 \mathrm{~Gy}$, and $74.67 \%$ at $0.2 \%$ EMS.

Whereas in combination treatments the highest percentage of mean seed yield per plant was noted $74.67 \%$ at $150 \mathrm{~Gy}+0.3 \% \mathrm{EMS}$, and followed by $65.45 \%$ at $200 \mathrm{GY}+0.3 \%$ EMS, respectively.

\section{Seedling height}

Mean seedling height (percentage) after 15 days of sowing was reported to be $82.27 \%$ at $150 \mathrm{~Gy}, 84.58 \%$ at $200 \mathrm{~Gy}, 94.60 \%$ at $400 \mathrm{~Gy}$, $76.56 \%$ at $0.2 \% \mathrm{EMS}, 92.75 \%$ at $0.3 \% \mathrm{EMS}$ and $58.37 \%$ at $0.4 \%$ EMS, respectively. 
Table.1 Various biological effects of gamma irradiation, EMS and their combinations in M1 generation

\begin{tabular}{|c|c|c|c|c|c|c|c|c|c|c|c|c|c|c|c|c|c|}
\hline \multirow[t]{2}{*}{ Variety } & \multirow[t]{2}{*}{ Doses } & \multicolumn{2}{|c|}{$\begin{array}{c}\text { Germination } \\
\%\end{array}$} & \multicolumn{2}{|c|}{$\begin{array}{l}\text { Plant height } \\
(\mathrm{cm}) \text { at } \\
\text { maturity }\end{array}$} & \multicolumn{2}{|c|}{$\begin{array}{c}\text { No. of } \\
\text { Pods/plant }\end{array}$} & \multicolumn{2}{|c|}{$\begin{array}{c}\text { No. of } \\
\text { seeds/plant }\end{array}$} & \multicolumn{2}{|c|}{$\begin{array}{l}\text { Seed yield/ } \\
\text { plant }(\mathrm{gm})\end{array}$} & \multicolumn{2}{|c|}{$\begin{array}{c}\text { Seedling } \\
\text { height }(\mathrm{cm}) \text { - } \\
\text { 15DAS }\end{array}$} & \multicolumn{2}{|c|}{$\begin{array}{l}100 \text { seed wt. } \\
(\mathrm{gm})\end{array}$} & \multicolumn{2}{|c|}{ Lethality } \\
\hline & & $\begin{array}{c}\text { Actual } \\
(\%)\end{array}$ & $\begin{array}{c}\% \text { of } \\
\text { control }\end{array}$ & $\begin{array}{l}\text { Actual } \\
(\%)\end{array}$ & $\begin{array}{l}\% \text { of } \\
\text { control }\end{array}$ & $\underset{\%}{\text { Actual }}$ & $\begin{array}{c}\% \text { of } \\
\text { control }\end{array}$ & $\begin{array}{c}\text { Actual } \\
(\%)\end{array}$ & $\begin{array}{c}\% \text { of } \\
\text { control }\end{array}$ & $\begin{array}{c}\text { Actual } \\
(\%)\end{array}$ & $\begin{array}{c}\% \text { of } \\
\text { control }\end{array}$ & $\begin{array}{l}\text { Actual } \\
(\%)\end{array}$ & $\begin{array}{l}\text { \% of } \\
\text { control }\end{array}$ & $\underset{\%}{\text { Actual }}$ & $\begin{array}{c}\% \text { of } \\
\text { control }\end{array}$ & $\begin{array}{c}\text { Actual } \\
(\%)\end{array}$ & $\begin{array}{c}\% \text { of } \\
\text { control }\end{array}$ \\
\hline \multirow{16}{*}{ JG 63} & Control & 87.33 & 100.00 & 54.33 & 100.00 & 64.67 & 100.00 & 41.00 & 100.00 & 6.70 & 100.00 & 12.97 & 100.00 & 16.93 & 100.00 & 32.67 & 100.00 \\
\hline & 150Gy & 73.67 & 84.36 & 51.00 & 93.87 & 58.33 & 90.20 & 31.00 & 75.61 & 4.23 & 63.13 & 6.20 & 47.80 & 14.30 & 84.47 & 46.33 & 38.60 \\
\hline & 200Gy & 63.00 & 72.14 & 39.00 & 71.78 & 43.33 & 67.00 & 25.67 & 62.61 & 5.73 & 85.52 & 5.61 & 43.25 & 15.20 & 89.78 & 57.00 & 47.49 \\
\hline & 400Gy & 56.33 & 64.50 & 43.67 & 80.38 & 39.33 & 60.82 & 22.33 & 54.46 & 4.03 & 60.15 & 2.97 & 22.90 & 13.73 & 81.10 & 63.67 & 53.05 \\
\hline & EMS 0.2\% & 60.00 & 68.70 & 45.00 & 82.83 & 25.67 & 39.69 & 30.67 & 74.80 & 5.10 & 76.12 & 9.80 & 75.56 & 13.17 & 77.79 & 60.00 & 49.99 \\
\hline & EMS 0.3\% & 52.67 & 60.31 & 41.00 & 75.46 & 43.00 & 66.49 & 16.80 & 40.98 & 4.57 & 68.21 & 8.00 & 61.68 & 12.97 & 76.61 & 67.33 & 56.10 \\
\hline & EMS 0.4\% & 46.00 & 52.67 & 44.00 & 80.99 & 32.67 & 50.52 & 33.00 & 80.49 & 3.67 & 54.78 & 6.70 & 51.66 & 13.67 & 80.74 & 74.00 & 61.66 \\
\hline & $\begin{array}{l}\text { 150Gy + 0.2\% } \\
\text { EMS }\end{array}$ & 58.00 & 66.41 & 46.67 & 85.90 & 49.00 & 75.77 & 21.67 & 52.85 & 2.53 & 37.76 & 11.33 & 87.36 & 12.40 & 73.24 & 62.00 & 51.66 \\
\hline & $\begin{array}{l}200 G y+0.2 \% \\
\text { EMS }\end{array}$ & 49.33 & 56.49 & 45.00 & 82.83 & 44.67 & 69.07 & 31.17 & 76.02 & 4.74 & 70.75 & 11.23 & 86.58 & 13.23 & 78.15 & 70.67 & 58.88 \\
\hline & $\begin{array}{l}\text { 150Gy + } 0.3 \% \\
\text { EMS }\end{array}$ & 44.67 & 51.15 & 45.33 & 83.43 & 38.00 & 58.76 & 25.33 & 61.78 & 4.43 & 66.12 & 9.73 & 75.02 & 13.30 & 78.56 & 75.33 & 62.76 \\
\hline & $\begin{array}{l}200 \mathrm{~Gy}+0.3 \% \\
\text { EMS }\end{array}$ & 33.67 & 38.55 & 43.00 & 79.15 & 34.00 & 52.57 & 34.67 & 84.56 & 4.47 & 66.72 & 6.33 & 48.80 & 12.53 & 74.01 & 86.33 & 71.93 \\
\hline & CV & 7.75 & & 4.30 & & 16.72 & & 7.17 & & 15.24 & & 11.83 & & 6.20 & & 6.96 & \\
\hline & CD $5 \%$ & 7.49 & & 3.32 & & 12.23 & & 3.48 & & 1.18 & & 2.06 & & 1.45 & & 7.49 & \\
\hline & CD $1 \%$ & 10.22 & & 4.53 & & 16.69 & & 4.74 & & 1.61 & & 2.81 & & 1.98 & & 10.22 & \\
\hline & S.Em.+ & 2.24 & & 1.12 & & 4.10 & & 1.18 & & 0.40 & & 0.69 & & 0.49 & & 2.54 & \\
\hline & F. cal & 33.12 & & 14.50 & & 7.38 & & 33.90 & & 7.18 & & 7.87 & & 7.11 & & 33.12 & \\
\hline
\end{tabular}


Table.2 Various biological effects of gamma irradiation, EMS and their combinations in M1 generation

\begin{tabular}{|c|c|c|c|c|c|c|c|c|c|c|c|c|c|c|c|c|c|}
\hline \multirow[t]{2}{*}{ Variety } & \multirow[t]{2}{*}{ Doses } & \multicolumn{2}{|c|}{ Germination \% } & \multicolumn{2}{|c|}{$\begin{array}{l}\text { Plant height } \\
(\mathrm{cm}) \text { at } \\
\text { maturity }\end{array}$} & \multicolumn{2}{|c|}{$\begin{array}{c}\text { No. of } \\
\text { Pods/plant }\end{array}$} & \multicolumn{2}{|c|}{$\begin{array}{c}\text { No. of } \\
\text { seeds/plant }\end{array}$} & \multicolumn{2}{|c|}{$\begin{array}{l}\text { Seed yield/ } \\
\text { plant (gm) }\end{array}$} & \multicolumn{2}{|c|}{$\begin{array}{c}\text { Seedling } \\
\text { height }(\mathrm{cm}) \text { - } \\
\text { 15DAS }\end{array}$} & \multicolumn{2}{|c|}{$\begin{array}{l}100 \text { seed wt. } \\
(\mathrm{gm})\end{array}$} & \multicolumn{2}{|c|}{ Lethality } \\
\hline & & $\begin{array}{c}\text { Actual } \\
(\%)\end{array}$ & $\begin{array}{c}\% \text { of } \\
\text { control }\end{array}$ & $\begin{array}{c}\text { Actual } \\
(\%)\end{array}$ & $\begin{array}{c}\% \text { of } \\
\text { control }\end{array}$ & $\begin{array}{c}\text { Actual } \\
\%\end{array}$ & $\begin{array}{c}\% \text { of } \\
\text { control }\end{array}$ & $\begin{array}{c}\text { Actual } \\
(\%)\end{array}$ & $\begin{array}{c}\% \text { of } \\
\text { control }\end{array}$ & $\begin{array}{c}\text { Actual } \\
(\%)\end{array}$ & $\begin{array}{c}\% \text { of } \\
\text { control }\end{array}$ & $\begin{array}{c}\text { Actual } \\
(\%)\end{array}$ & $\begin{array}{c}\% \text { of } \\
\text { control }\end{array}$ & $\begin{array}{c}\text { Actual } \\
\%\end{array}$ & $\begin{array}{c}\% \text { of } \\
\text { control }\end{array}$ & $\begin{array}{c}\text { Actual } \\
(\%)\end{array}$ & $\begin{array}{c}\% \text { of } \\
\text { control }\end{array}$ \\
\hline \multirow{16}{*}{ JG 74} & Control & 78 & 100.00 & 53 & 100 & 62.67 & 100 & 46.67 & 100 & 5.97 & 100 & 13.2 & 100 & 16.93 & 100 & 29.33 & 100 \\
\hline & 150Gy & 66 & 84.62 & 33.33 & 62.89 & 57 & 90.95 & 42 & 89.99 & 4.93 & 82.58 & 10.17 & 77.05 & 13.33 & 78.74 & 66 & 55.00 \\
\hline & 200Gy & 50.33 & 64.53 & 31.33 & 59.11 & 35.33 & 56.37 & 34.67 & 74.29 & 4.03 & 67.50 & 12.8 & 96.97 & 13.97 & 82.52 & 50.33 & 41.94 \\
\hline & 400Gy & 41.67 & 53.42 & 34.67 & 65.42 & 33.33 & 53.18 & 19 & 40.71 & 3.14 & 52.60 & 10.87 & 82.35 & 12.73 & 75.19 & 41.67 & 34.72 \\
\hline & EMS 0.2\% & 52.33 & 67.09 & 48 & 90.57 & 25.67 & 40.96 & 45.67 & 97.86 & 4.97 & 83.25 & 9.8 & 74.24 & 13.03 & 76.96 & 52.33 & 43.61 \\
\hline & EMS 0.3\% & 44 & 56.41 & 48.33 & 91.19 & 34.33 & 54.78 & 17 & 36.43 & 4.47 & 74.87 & 10.07 & 76.29 & 15.33 & 90.55 & 44 & 36.66 \\
\hline & EMS 0.4\% & 42.33 & 54.27 & 47.73 & 90.06 & 27.33 & 43.61 & 29.67 & 63.57 & 3.93 & 65.83 & 11.17 & 84.62 & 15.17 & 89.60 & 42.44 & 35.36 \\
\hline & $\begin{array}{l}\text { 150Gy }+ \\
0.2 \% \text { EMS }\end{array}$ & 54.67 & 70.09 & 43.73 & 82.51 & 49 & 78.19 & 20 & 42.85 & 4.27 & 71.52 & 11.03 & 83.56 & 15.7 & 92.73 & 54.67 & 45.56 \\
\hline & $\begin{array}{l}200 G y+ \\
0.2 \% \text { EMS }\end{array}$ & 46 & 58.97 & 50.33 & 94.96 & 40.33 & 64.35 & 41.67 & 89.29 & 3.8 & 63.65 & 11.5 & 87.12 & 13.83 & 81.69 & 46 & 38.33 \\
\hline & $\begin{array}{l}\text { 150Gy }+ \\
0.3 \% \text { EMS }\end{array}$ & 37 & 47.44 & 43.2 & 81.51 & 34.67 & 55.32 & 25.33 & 54.27 & 3.63 & 60.80 & 12.17 & 92.20 & 13.07 & 77.20 & 37 & 30.83 \\
\hline & $\begin{array}{l}200 \mathrm{~Gy}+ \\
0.3 \% \text { EMS }\end{array}$ & 31 & 39.74 & 42.13 & 79.49 & 30.67 & 48.94 & 42 & 89.99 & 4.2 & 70.35 & 10.37 & 78.56 & 13.73 & 81.10 & 31 & 25.83 \\
\hline & CV & 7.75 & & 10.1 & & 18.02 & & 32.03 & & 11.83 & & 7.23 & & 6.2 & & 9.05 & \\
\hline & CD $5 \%$ & 7.49 & & 7.44 & & 12.01 & & 17.63 & & 0.87 & & 1.37 & & 1.45 & & 6.93 & \\
\hline & CD 1\% & 10.22 & & 10.15 & & 16.38 & & 24.06 & & 1.1 & & 1.88 & & 1.98 & & 9.4 & \\
\hline & S.Em.+ & 2.5 & & 2.52 & & 4.07 & & 5.9 & & 0.29 & & 0.46 & & 0.49 & & 2.3 & \\
\hline & F. cal & 33.22 & & 8.32 & & 8.78 & & 4.03 & & 6.78 & & 5.83 & & 7.11 & & 20.66 & \\
\hline
\end{tabular}


Table.3 Various biological effects of gamma irradiation, EMS and their combinations in M1 generation

\begin{tabular}{|c|c|c|c|c|c|c|c|c|c|c|c|c|c|c|c|c|c|}
\hline \multirow[t]{2}{*}{ Variety } & \multirow[t]{2}{*}{ Doses } & \multicolumn{2}{|c|}{ Germination \% } & \multicolumn{2}{|c|}{$\begin{array}{l}\text { Plant height } \\
(\mathrm{cm}) \text { at maturity }\end{array}$} & \multicolumn{2}{|c|}{$\begin{array}{c}\text { No. of } \\
\text { Pods/plant }\end{array}$} & \multicolumn{2}{|c|}{$\begin{array}{c}\text { No. of } \\
\text { Seeds/plant }\end{array}$} & \multicolumn{2}{|c|}{$\begin{array}{l}\text { Seed yield/ } \\
\text { plant }(\mathrm{gm})\end{array}$} & \multicolumn{2}{|c|}{$\begin{array}{l}\text { Seedling height } \\
(\mathrm{cm}) \text {-15DAS }\end{array}$} & \multicolumn{2}{|c|}{$\begin{array}{l}100 \text { Seed wt. } \\
(\mathrm{gm})\end{array}$} & \multicolumn{2}{|c|}{ Lethality } \\
\hline & & $\begin{array}{c}\text { Actual } \\
(\%)\end{array}$ & $\begin{array}{l}\% \text { of } \\
\text { control }\end{array}$ & $\begin{array}{l}\text { Actual } \\
(\%)\end{array}$ & $\begin{array}{l}\% \text { of } \\
\text { control }\end{array}$ & $\begin{array}{c}\text { Actual } \\
\%\end{array}$ & $\begin{array}{c}\% \text { of } \\
\text { control }\end{array}$ & $\begin{array}{c}\text { Actual } \\
(\%)\end{array}$ & $\begin{array}{c}\% \text { of } \\
\text { control }\end{array}$ & $\begin{array}{c}\text { Actual } \\
(\%)\end{array}$ & $\begin{array}{c}\% \text { of } \\
\text { control }\end{array}$ & $\begin{array}{l}\text { Actual } \\
(\%)\end{array}$ & $\begin{array}{c}\% \text { of } \\
\text { control }\end{array}$ & $\begin{array}{c}\text { Actual } \\
\%\end{array}$ & $\begin{array}{l}\% \text { of } \\
\text { control }\end{array}$ & $\begin{array}{c}\text { Actual } \\
(\%)\end{array}$ & $\begin{array}{c}\% \text { of } \\
\text { control }\end{array}$ \\
\hline \multirow{16}{*}{ JG 130} & Control & 87 & 100 & 50.67 & 100 & 49.67 & 100 & 37 & 100 & 6.83 & 100 & 12.97 & 100 & 18.93 & 100 & 33 & 100 \\
\hline & 150Gy & 61 & 70.11 & 39.67 & 78.29 & 30.67 & 61.75 & 21.43 & & 4.23 & 61.93 & 10.67 & 82.27 & 16.87 & 89.12 & 59 & 49.17 \\
\hline & 200Gy & 53 & 60.92 & 34.83 & 68.74 & 14 & 28.19 & 20 & 54.05 & 5.03 & 73.65 & 10.97 & 84.58 & 15.93 & 84.15 & 67 & 55.83 \\
\hline & 400Gy & 49 & 56.32 & 34 & 67.10 & 17.67 & 35.57 & 21.67 & 58.57 & 4.03 & 59.00 & 12.27 & 94.60 & 14.47 & 76.44 & 70.67 & 58.89 \\
\hline & EMS 0.2\% & 51 & 58.62 & 42.67 & 84.21 & 13.67 & 27.52 & 31.67 & 85.59 & 5.1 & 74.67 & 9.93 & 76.56 & 16.8 & 88.75 & 69 & 57.50 \\
\hline & EMS 0.3\% & 43 & 49.43 & 46.97 & 92.70 & 27.33 & 55.02 & 19.67 & 53.16 & 4.57 & 66.91 & 12.03 & 92.75 & 15.83 & 83.62 & 77 & 64.17 \\
\hline & EMS 0.4\% & 38 & 43.68 & 42.57 & 84.01 & 30.67 & 61.75 & 23.73 & 64.14 & 3.93 & 57.54 & 7.57 & 58.37 & 14.64 & 77.34 & 82 & 68.33 \\
\hline & $\begin{array}{l}150 \mathrm{~Gy}+0.2 \% \\
\text { EMS }\end{array}$ & 51 & 58.62 & 44.67 & 88.16 & 42.33 & 85.22 & 25.4 & 68.65 & 4.27 & 62.52 & 10.1 & 77.87 & 16.07 & 84.89 & 68.67 & 57.23 \\
\hline & $\begin{array}{l}200 G y+0.2 \% \\
\text { EMS }\end{array}$ & 42 & 48.28 & 44 & 86.84 & 40.67 & 81.88 & 26.7 & 72.16 & 3.8 & 55.64 & 9.89 & 76.25 & 15.63 & 82.57 & 78 & 65.00 \\
\hline & $\begin{array}{l}\text { 150Gy + 0.3\% } \\
\text { EMS }\end{array}$ & 39 & 44.83 & 37.13 & 73.28 & 36.33 & 73.14 & 19.2 & 51.89 & 5.1 & 74.67 & 9.73 & 75.02 & 14.87 & 78.55 & 81 & 67.50 \\
\hline & $\begin{array}{l}200 \mathrm{~Gy}+ \\
0.3 \% \text { EMS }\end{array}$ & 29 & 33.33 & 35.33 & 69.73 & 25.67 & 51.68 & 33.67 & 91.00 & 4.47 & 65.45 & 6.33 & 48.80 & 14.63 & 77.28 & 91 & 75.83 \\
\hline & CV & 6.6 & & 8.46 & & 16.72 & & 21.92 & & 11.27 & & 11.83 & & 6.09 & & 6.96 & \\
\hline & CD 5\% & 5.52 & & 5.93 & & 12.23 & & 9.4 & & 0.89 & & 2.06 & & 1.64 & & 7.49 & \\
\hline & CD $1 \%$ & 7.6 & & 8.08 & & 16.69 & & 12.9 & & 1.22 & & 2.8 & & 2.24 & & 10.22 & \\
\hline & S.Em.+ & 1.8 & & 2.01 & & 4.14 & & 3.3 & & 0.3 & & 0.6 & & 0.55 & & 2.5 & \\
\hline & F. cal & 64.76 & & 7.23 & & 7.38 & & 3.37 & & 7.9 & & 7.87 & & 5.56 & & 33.2 & \\
\hline
\end{tabular}


Whereas, in combination treatments the mean seedling height was noted to be $77.87 \%$ at $150 \mathrm{~Gy}+0.2 \% \mathrm{EMS}, 76.25 \%$ at $200 \mathrm{~Gy}+0.2 \%$ EMS, $75.02 \%$ at $150 \mathrm{~Gy}+0.3 \%$ EMS and $48.80 \%$ at $200 \mathrm{~Gy}+0.3 \% \mathrm{EMS}$ respectively, as compared to control.

\section{0 seed weight}

The mean 100 seed weight percentage was reported to be $89.12 \%$ at $150 \mathrm{~Gy}, 84.15 \%$ at $200 \mathrm{~Gy}$ and $76.44 \%$ at $400 \mathrm{~Gy}$ respectively. Whereas in chemical mutagenic treatments it was recorded to be $88.75 \%$ at $0.2 \%$ EMS, $83.62 \%$ at $0.3 \%$ EMS and $77.34 \%$ at $0.4 \%$ EMS also in combination treatments the mean 100 seed weight percentage was noted to be 84.89 at $150 \mathrm{~Gy}+0.2 \% \mathrm{EMS}, 82.57 \%$ at 200 $\mathrm{Gy}+0.2 \%$ EMS, $78.55 \%$ at $150 \mathrm{~Gy}+0.3 \%$ EMS and $77.28 \%$ at $200 \mathrm{~Gy}+0.3 \%$ EMS respectively, as compared to control.

\section{Lethality}

The highest lethality percentage was recorded in physical mutagenic treatments, which was $58.89 \%$ at $400 \mathrm{~Gy}$, whereas in chemical mutagenic treatment it was $68.33 \%$ at $0.4 \%$ EMS. Similarly in combination treatments the maximum lethality percentage was recorded to be $75.83 \%$ in $200 \mathrm{~Gy}+0.3 \%$ EMS as compared to control.

\section{The impact of the mutagenic treatments on seed germination and on some morphological traits}

From the present investigation it was revealed that the mutagenic treatments viz., 200Gy, 400Gy, $\quad 0.3 \% \quad$ EMS, $0.4 \%$ EMS, $150 \mathrm{~Gy}+0.3 \% \mathrm{EMS}$ and $200 \mathrm{~Gy}+0.3 \% \mathrm{EMS}$ has found to shown some prominent effect on individuals of selected chickpea varieties viz., JG 63, JG 74 and JG 130 as compared to other treatments. There is a change in terms of decrease in seedling height, germination percentage, plant height, number of pods per plant, number of seeds per plant and seed yield per plant as compared to control. Whereas, similar experiment conducted by [3] against the ICL 6104 and ICL 3279 genotypes. They found the similar changes in some biological parameters like seedling height, germination and plant height. [4] observed decrease in plant height, number of primary and secondary branches, pods per plant, seeds per pod and grain yield in $\mathbf{M}_{2}$ generation were significantly affected due to genotypes, treatments and also by their interaction. Similar kinds of results were obtained in the present investigation on $\mathrm{M}_{1}$ generation. [5] Observed reduced germination percentage at higher dosages in all lines, especially in induced mutant lines of ILC 5901.

In the present study, the germination percentage decreases by applying higher doses of chemical and physical mutagens. As discussing about comparative mutagenic effectiveness, it was found that EMS treatments are more efficient than gamma radiation according to the biological and agronimical parameters, as similar with the findings of [6]. It was also evident from the present study that the effectiveness of EMS treatments initially increased with an increase in concentration but decreased at higher concentration. The combination of $200 \mathrm{~Gy}+$ $0.3 \%$ EMS treatment was found most effective in the populations of all three genotypes. The present findings are conformity with the findings of [7], [8] and [9].

The overall trend of effectiveness of mutagenic treatment on experimental genotypes, JG 63 and JG 74 showed changes in their varietal characteristics are in agreement with the findings of [6] in case of $\mathrm{Pb}-2000$ and $\mathrm{C}-44$ with mutagenic treatments.

\section{References}


1. Micke A. 2004. Genetic improvement of grain legumes using induced mutations. An overview. In Improvement of grain legume production using induced mutations. IAEA, Vienna (1):50-51.

2. Maluszynski M, Van Zanten 1, Asir A, Brunner H, Ahloowalia B, Zapata FJ, Weck E, (2005). Mutation techniques in plant breeding. Proc. induced mutations and molecular techniques for crop improvement. Fao/iaea, Austria, Vienna, June (19-23): 489-504.

3. Haq MA, Singh K, Abidin $Z$ and Ahmad MS. 2003. Mutation studies in chickpea (Cicer arietinum L.) Pak. J. Agri. Sci., Vol. 29, No.4.

4. Khan S, Wani MR, Bhat M and Kouser P. 2005. Induced chlorophyll mutations in chickpea (Cicer arietinum L.) Iinternational journal of agriculture and biology 1560-8530/2005/07-5-764767

5. Omar $M$ and singh KB. 2006. Development of early mutants with resistance to ascochyta blight or leaf miner. International Chickpea and Pigeonpea Newsletter 1995 No. 2 pp. 10-11.

6. Shah T, Mirza J, and Haq MA and Babar MA. 2008 Induced genetic variability in chickpea (Cicer arientinum L.) and comparative mutagenic effectiveness and efficiency of physical and chemical mutagens. pak. J. Bot., 40(2): 605-613.

7. Kharakwal MC. 2003 Induced mutations in chickpea (Cicer arietinum L.) VI.Significance of Induced altered correlations, Indian J. Genet. 63(3): 219-224.

8. Aijaz A. Wani 2009. Mutagenic Effectiveness and efficiency of Gamma Rays, Ethyl Methane sulphonate and their combination treatment in chickpea (Cicer arietinum L.) Asian Journal of Plant Sciences 8(4): 318-321.

9. Bashir S, Wani A and Nawchoo I. 2013. Studies on mutagenic effectiveness and efficiency in Fenugreek (Trigonella foenum-graecum L.) African Journal of Biotechnology Vol. 12(18), pp. 24372440.

\section{How to cite this article:}

Maruti Pawar, O.M. Gupta and Devashish Chobe. 2018. Radiation Induced Mutation for Resistance against Dry Root Rot in Chickpea (Cicer arietinum Linn.). Int.J.Curr.Microbiol.App.Sci. 7(07): 3542-3551. doi: https://doi.org/10.20546/ijcmas.2018.707.411 